\title{
Teufelsabbiss, Blume des Jahres 2015
}

\author{
Armin Jagel
}

\begin{abstract}
Devil's bit (Succisa pratensis) is flower of the year 2015. Its biology, mythology and medicinal uses are outlined.
\end{abstract}

\section{Zusammenfassung}

Der Teufelsabbiss (Succisa pratensis) ist Blume des Jahres 2015. Biologie, Mythologie und medizinische Verwendungen werden erläutert. Ergänzend werden einige Dipsacus-Arten, enge Verwandte des Teufelsabbisses, und ihr Aufblühmodus vorgestellt.

\section{Einführung}

Schon der Name Teufelsabbiss lässt auf mythische Geschichten schließen, die mit der Pflanze verbunden werden. Früher war der Teufelsabbiss (Succisa pratensis) samt seiner Heilwirkungen viel bekannter als heute. Gründe dafür mögen sein, dass diese Wildstaude viel seltener geworden ist und oft nur noch in Schutzgebieten wächst, wo sie nicht gepflückt werden darf. Die Wahl zur Blume des Jahres ist gut begründet, weil sie auf den starken Rückgang seines
Lebensraumes, also Feuchtwiesen, Moor- und Heidegebiete sowie intakte Uferzonen und Gräben im Feuchtgrünland, hinweist.

\section{Succisa pratensis und der Teufel}

Früher stand der Teufelsabbiss (Satanswurz, Teufelswurz) wegen seiner heilsamen Wurzel als Medizinalpflanze in hohem Ansehen (DüLL

Abb. 1: Succisa pratensis auf einer feuchten Brache in Gütersloh.

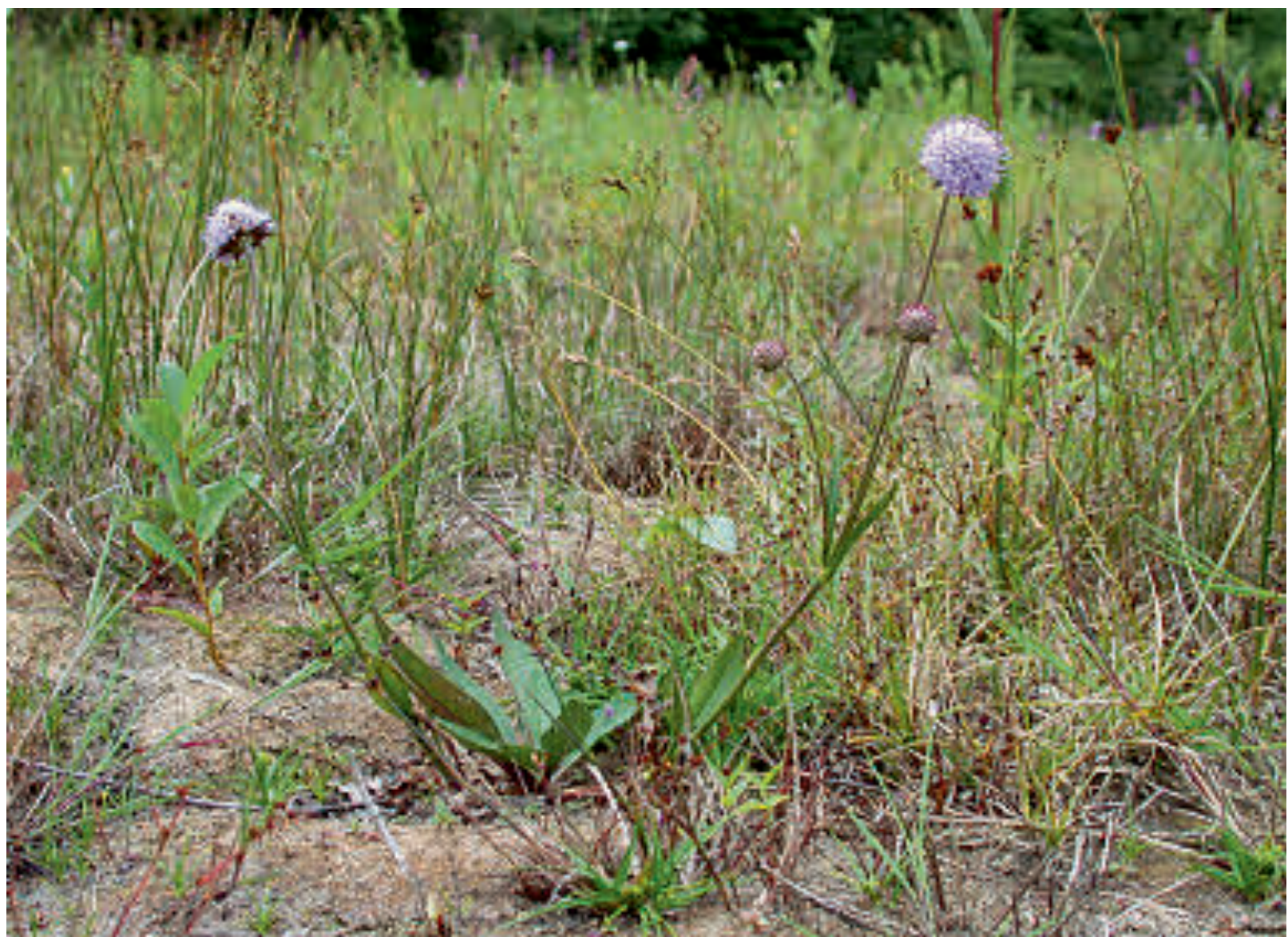



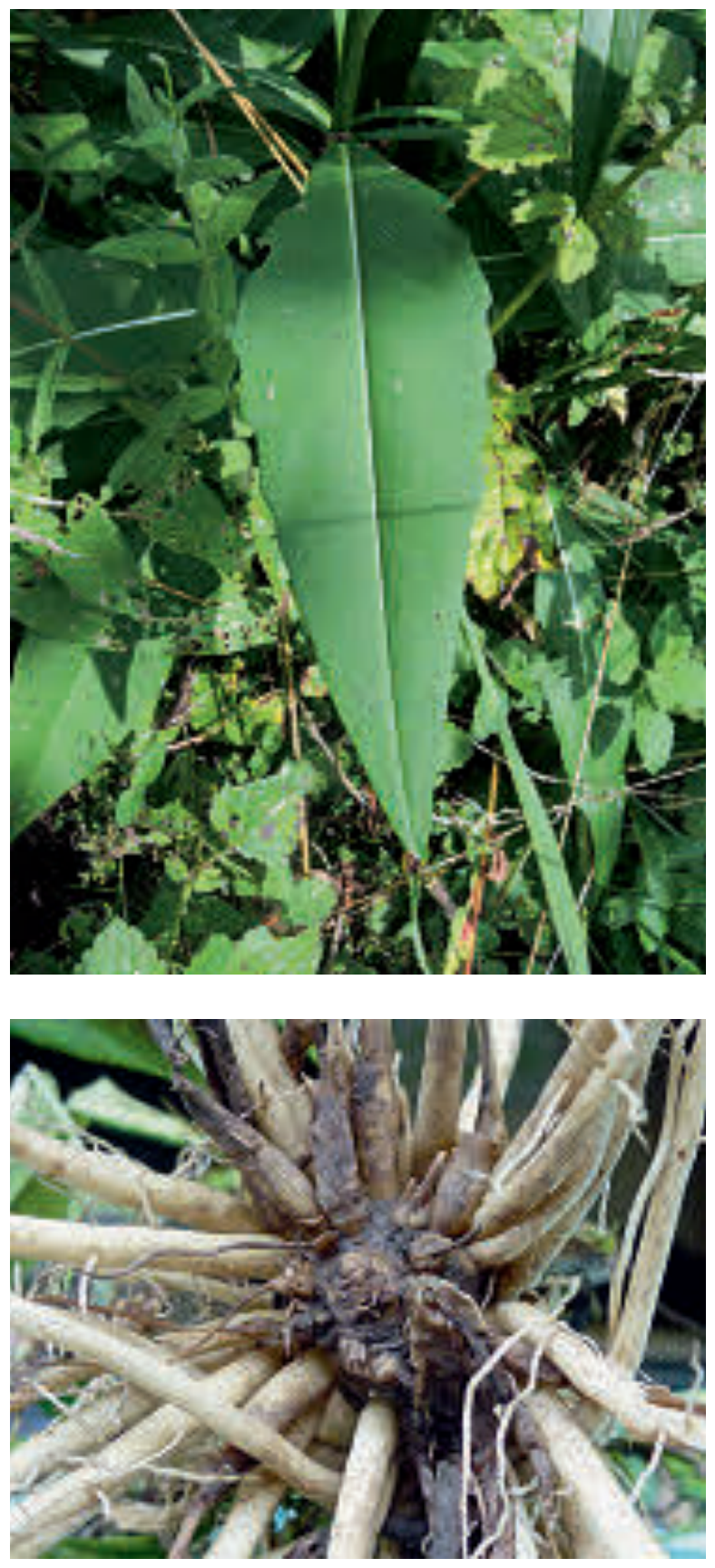

\& Kutzelnigg 2011). Nach altem Glauben gönnt der Teufel dem Menschen aber die Nutzung der Wurzel nicht, weil sie eine kraftvolle Heilwirkung besitzt, und weil alles, was gut ist, vom Teufel verachtet wird. Deswegen beißt er sie ab, sobald sie ausgewachsen ist. Dieser Mythos beruht auf der Beobachtung, dass die Hauptwurzel der Pflanze abfault und dann unten wie abgeschnitten (= lat. succisus) oder eben abgebissen erscheint. Der Teufel beißt die Wurzel angeblich erst am Johannistag ab, dann aber so schnell wie der Blitz und überall gleichzeitig.
Wer die heilkräftige Wurzel sammeln möchte, muss dies deshalb genau um Mitternacht, direkt vor dem Johannistag, tun. Der Besitz der Wurzel hatte nach altem Aberglauben einen hochgeschätzten Nutzen, denn sie bannte den Teufel und schützte vor Hexen. Der Teufelsabbiss sollte aber auch gegen verschiedene Krankheiten helfen. So hängte man sich zerkleinerte Wurzeln um den Hals, die volksmedizinisch beim Trocknen Augenleiden heilen sollten.

In der Steiermark wurde Teufelsabbiss als Heildroge in der Apotheke angeboten. Wenn ein Tier im Stall verendete, mischte man dort Teile der Pflanze zusammen mit Salz unter das Futter und schützte damit das verbliebene Vieh im Stall (Haerkötter \& Haerkötter 1987, SсHÖpF 1992).

Heute gibt es offenbar wirksamere Mittel gegen Teufel und Hexen. In vielen neueren Heilbüchern wird der Teufelsabbiss nicht mehr aufgeführt. Die Schulmedizin sieht noch reichlich Forschungsbedarf hinsichtlich einer möglichen Heilwirkung, sodass die Droge des Teufelsabbisses (Morsus diaboli) nur in der Volksheilkunde genutzt wird. Sie enthält u. a. Saponine, Bitter- und Gerbstoffe. Man verwendet sie zur sogenannten Blutreinigung, als wassertreibendes Mittel (Diuretikum), bei Husten, Heiserkeit und Lungenkrankheiten sowie als Wurmmittel. Äußerlich angewendet soll Teufelsabbiss gegen Hauterkrankungen und Quetschungen helfen. Diese Anwendungen des Teufelsabbis-

Abb. 2 (oben): Laubblatt von Succisa pratensis.

Abb. 3 (unten): Wurzeln des Teufelsabbisses von unten, Narbe der abgefaulten Hauptwurzel erkennbar.

Abb. 4 (Seite 99 oben links): Junger Blütenstand des Teufelsabbisses mit Schwebfliege. Der Blütenstand blüht von oben und unten auf.

Abb. 5 (Seite 99 oben rechts): Kopfiger, voll aufgeblühter Blütenstand von Succisa pratensis im Hasselbachtal in Hagen.

Abb. 6 (Seite 99 unten links): Dipsacus fullonum. Hier öffnen sich die ersten Blüten in der Mitte des Blütenstandes.

Abb. 7 (Seite 99 unten rechts): Dipsacus laciniatus. Die geöffneten Blüten stehen in zwei Ringen am Blütenstand. 

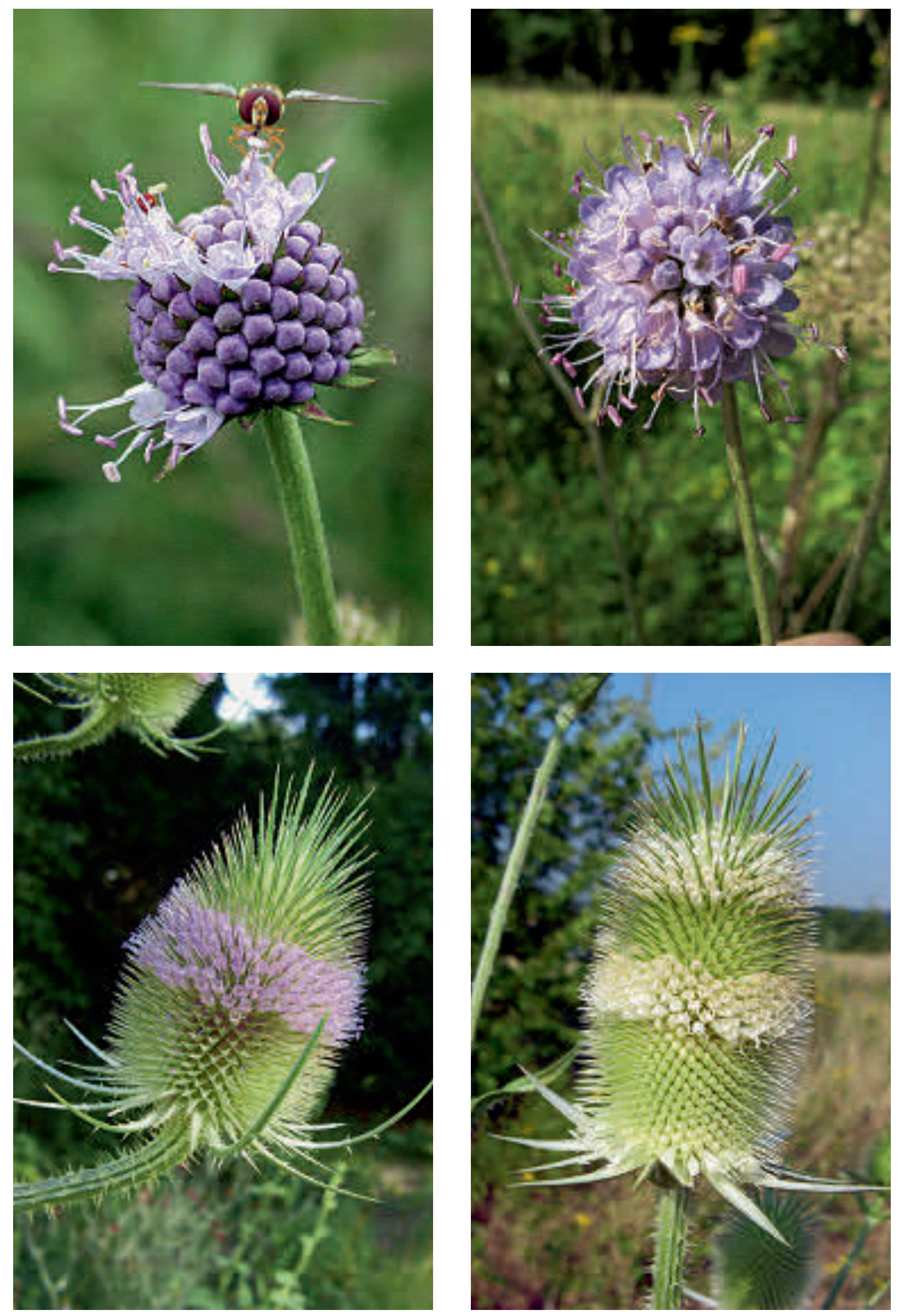

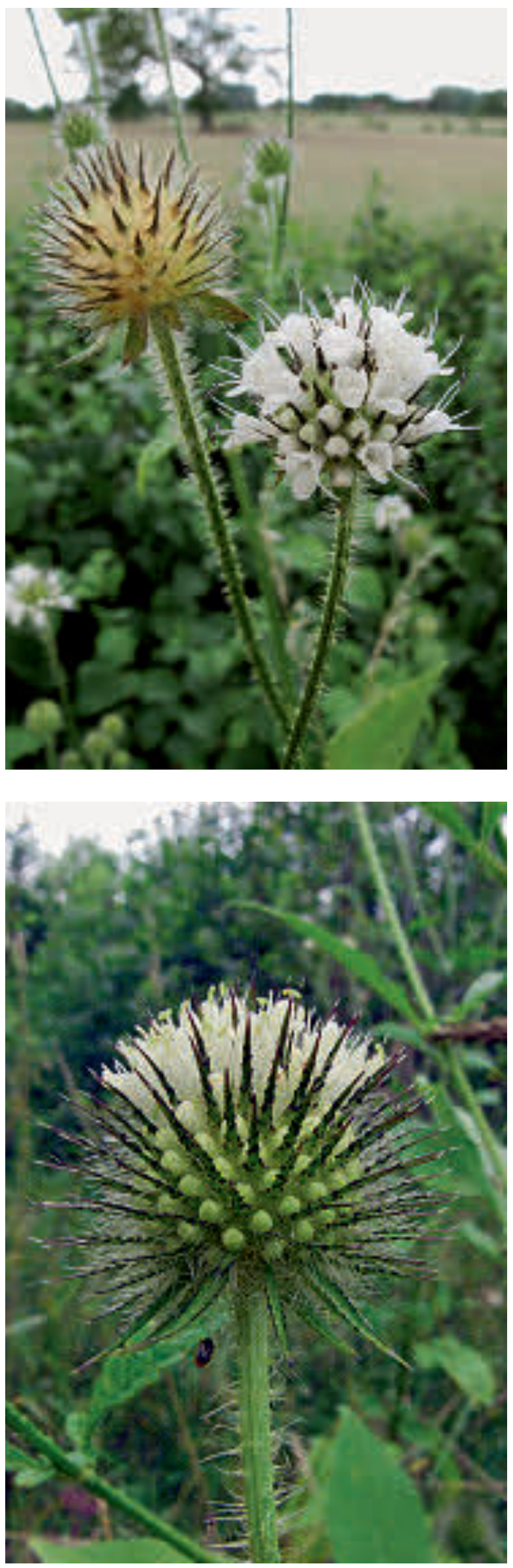

ses wurden schon im Mittelalter genutzt (Pahlow 1993, Hiller \& Melzig 2002).

\section{Blüten}

Die ungestielten Blüten des Teufelsabbisses sind meist blauviolett und in rundlichen Köpfchen vereinigt. Sie haben eine verwachsene Blütenkrone mit vier Zipfeln. Die Blütenstände sind von einer Hochblatthülle umgeben und ähneln daher denen von Korbblütlern. Anders als bei diesen ist beim Teufelsabbiss aber z. B. ein Außenkelch vorhanden und sind die Staubblätter nicht miteinander verwachsen.

Die Blüten sind vormännlich. In jeder von ihnen werden zuerst die Staubblätter reif, dann erst die Narben, was der Vermeidung von Selbstbestäubung dient. Die Staubbeutel sind deutlich schwächer blau gefärbt als die Blütenblätter. Dieser Kontrast ist für die Bestäuber für das Auffinden des Pollens wichtig. Neben Pflanzen mit zwittrigen Blüten kommen auch solche mit ausschließlich weiblichen Blüten vor (Düll \& Kutzelnigg 2011). Die Blütezeit des Teufelsabbisses fällt in die Monate (Juni) Juli bis September und liegt damit relativ spät im Jahr. Bestäubt werden die Blüten bei uns vor allem von Honig- und Wildbienen, Hummeln, Fliegen und 28 verschiedenen Schmetterlingsarten (Hintermeier \& Hintermeier 2012).

\section{Verwandtschaft}

Der Teufelsabbiss gehört zu den Kardengewächsen (Dipsacaceae). Die Verwandtschaft mit Acker-Witwenblume (Knautia arvensis), Wald-Witwenblume (Knautia dipsacifolia) und Tauben-Skabiose (Scabiosa columbaria) ist offensichtlich, allerdings fehlen dem Teufelsabbiss die vergrößerten Randblüten.

\section{Abb. 8 (oben): Dipsacus pilosus mit Blüten- und} Fruchtstand.

Abb. 9 (unten): Dipsacus strigosus. Das Aufblühen beginnt an der Spitze des Blütenstandes.

Abb. 10 (Seite 101 oben): Teufelsabbiss mit Acker-Hummel (Bombus pascuorum).

Abb. 11 (Seite 101 unten): Darstellung einer Teufelsabbiss-Pflanze im Kräuterbuch des LeONHART Fuchs von 1543. 
Ein Hinweis auf die Verwandtschaft des Teufelsabbisses mit der Wilden Karde (Dipsacus fullonum) ist eine ungewöhnliche Aufblühreihenfolge im Blütenstand. Bei Pflanzen mit offenen Blütenständen blüht dieser von unten nach oben auf, bei flachen Köpfchen der Korbblütler (wie z.B. dem Gänseblümchen) dementsprechend von außen nach innen. Bei der Wilden Karde beginnt das Aufblühen des Blütenstandes im mittleren Bereich mit einem Ring von Blüten. Anschließend erscheinen zwei Blühringe, von denen einer nach oben hin und der andere nach unten hin aufblüht.

Zumindest bei anderen Karden kommen aber auch andere Varianten vor, wie bei der Schlitzblättrigen (Dipsacus laciniatus) und bei der Schlanken Karde (Dipsacus strigosus). Im Falle der Behaarten Karde (Dipsacus pilosus) treten offensichtlich ganz unregelmäßige Aufblühfolgen auf. Welche Varianten bei der Aufblühfolge beim Teufelsabbiss existieren, ist offenbar noch gar nicht geklärt, jedenfalls variiert sie auch hier. Solche Phänomene sind am besten durch Untersuchungen an sehr jungen Stadien mit dem Rasterelektronenmikroskop zu lösen, welche offenbar bisher nicht vorliegen.

\section{Dank}

Für die Bereitstellung von Bildern bedanke ich mich herzlich bei Annette Höggemeier (Witten) und Veit Martin Dörken (Konstanz).

\section{Literatur}

Düll, R. \& Kutzelnigg, H. 2011: Taschenlexikon der Pflanzen Deutschlands und angrenzender Länder, 7. Aufl. - Wiebelsheim.

Haerkötter, G. \& Haerkötter, M. 1987: Hexenfurz und Teufelsdreck. Liebes-, Heil- und Giftkräuter: Hexereien, Rezepte und Geschichten. - Hamburg. Hiller, K. \& Melzig, M. F. 2003: Lexikon der Arzneipflanzen und Drogen. - Heidelberg, Berlin. Hintermeier, H. \& Hintermeier, M. 2012: Blütenpflanzen und ihre Gäste, Teil 3. - Bad Windsheim.

Pahlow, M. 1993: Das große Buch der Heilpflanzen. - München.

Sснӧрғ, H. 1992: Zauberkräuter. - Graz.

\section{Internetseite}

Loki-Schmidt-Stiftung 2014: Der Gewöhnliche Teufelsabbiss ist die Blume des Jahres 2015.

- http://www.loki-schmidt-stiftung.de/downloads/

blumen_des_jahres_pdfs/Blume_des_Jahres_2015.pdf
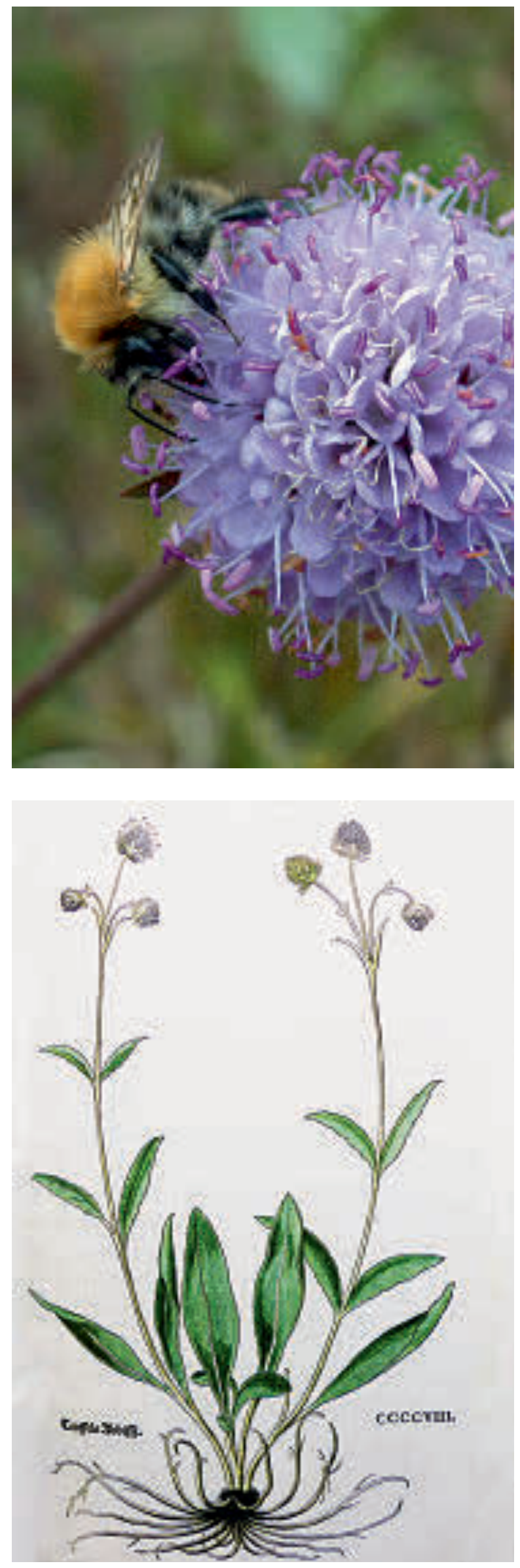